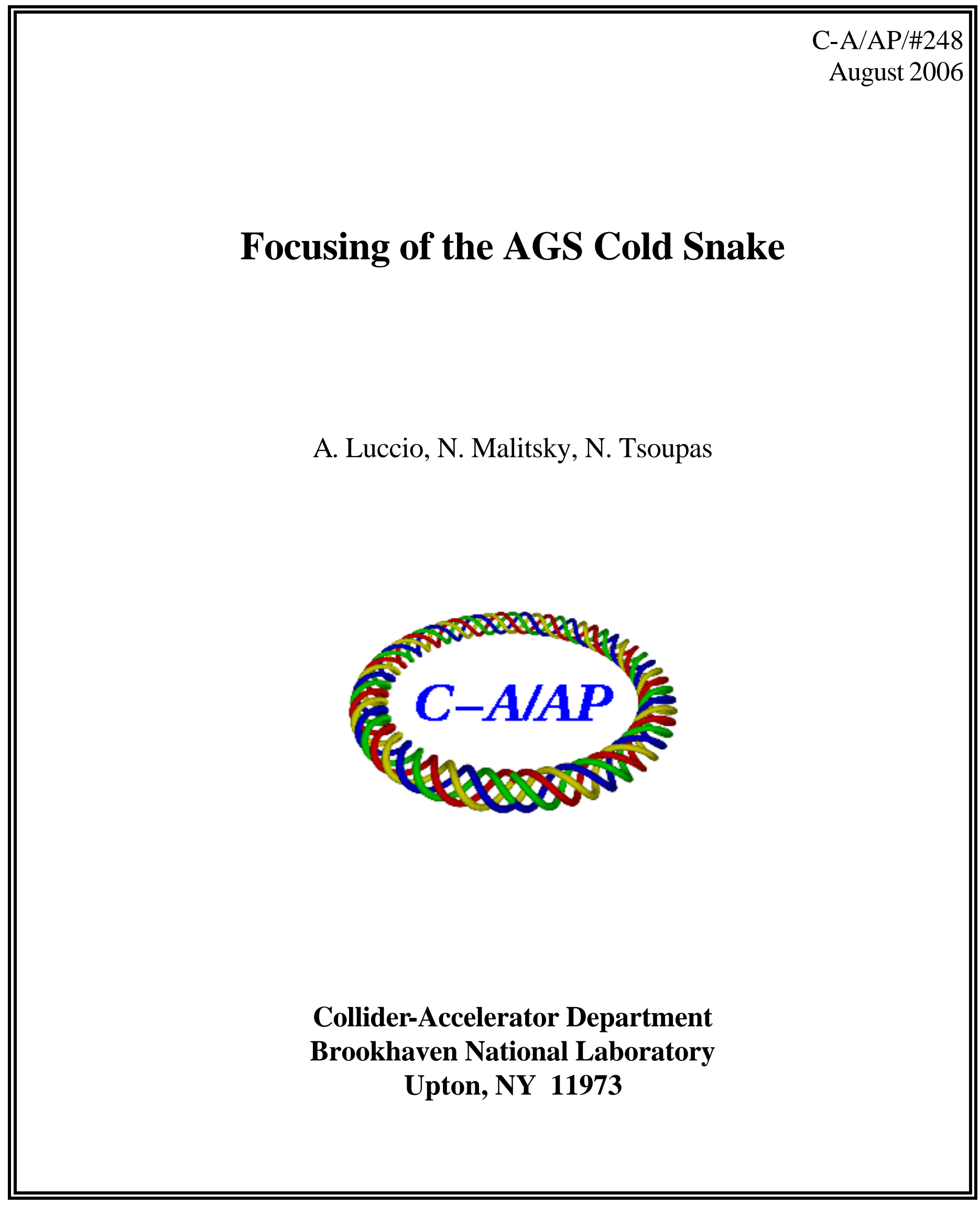




\title{
Focusing of the AGS Cold Snake
}

\author{
A.U.Luccio, N.Malitsky, N.Tsoupas
}

July 28,2006

We know that the superconductive helical partial snake for the AGS[1] is focusing in both planes. Also, in the vertical plane the field integrals are well compensated, so, a particle entering the snake straight on axis emerges (almost) straight on axis. In vertical, the orbit is well balanced, i.e its maximum excursion up is equal to its maximum excursion down.

The situation is different in the horizontal plane. A particle entering straight on axis emerges at some non zero angle and the orbit excursion is not balanced with respect to the snake axis. The situation is shown in Fig. 1 and Fig. 2 for the case of a cold snake with field of 2 Tesla.

In the horizontal plane we can choose to offset the orbit at the entrance with a magnetic bump in a way to center the orbit displacement in the snake, or to offset the orbit in order to obtain a zero angle of the trajectory at snake's exit. For the present example, the first case asks for an orbit offset of $\approx-22 \mathrm{~mm}$, and the second case requires an orbit offset of $\approx-16 \mathrm{~mm}$. In the first case the horizontal orbit angle at the exit of the snake is of $\approx 5 \mathrm{mrad}$, that must be compensated by some horizontal steering in the bump used to produce the offset.

In the vertical plane the angle of the orbit for a central particle is $\approx 0.08 \mathrm{mrad}$ at a beam energy corresponding to $\gamma=2.5$.

As the figures show, the focusing of the snake in both planes is also a function of the orbit offset at the entrance, since the snake contains a substantial amount of higher order multipoles.

The focusing of the snake for various x-offset values is shown in Fig.3. The effect is large for the horizontal focusing, with a focal legth changing by almost a factor 3 over the range considered. As expected, we only see a small focus variation generated by a range of orbit y-offsets.

To model the snake as a machine element to be used, e.g., in $M A D$, we have calculated symplectic transfer matrices and second order maps for the 6 dimension canonical phase space vector $\left(x, p_{x}, y, p_{y},-c \Delta t, \Delta E / p c\right)$. These maps were obtained as a first and second order Jacobian for the transformation between entrance and exit of the snake, with orbits calculated with the code Snig [2] by integration through a numerical map of the snake field provided by S.Gupta. The snakes maps that have been used in $M A D$ for modelling of the AGS with snakes correspond to the case for the horizontal orbit balanced [3].

Without further elaboration, we show in Figs.4, 5, 6, and 7 the horizontal focusing pattern for other values of the snake magnetic field 


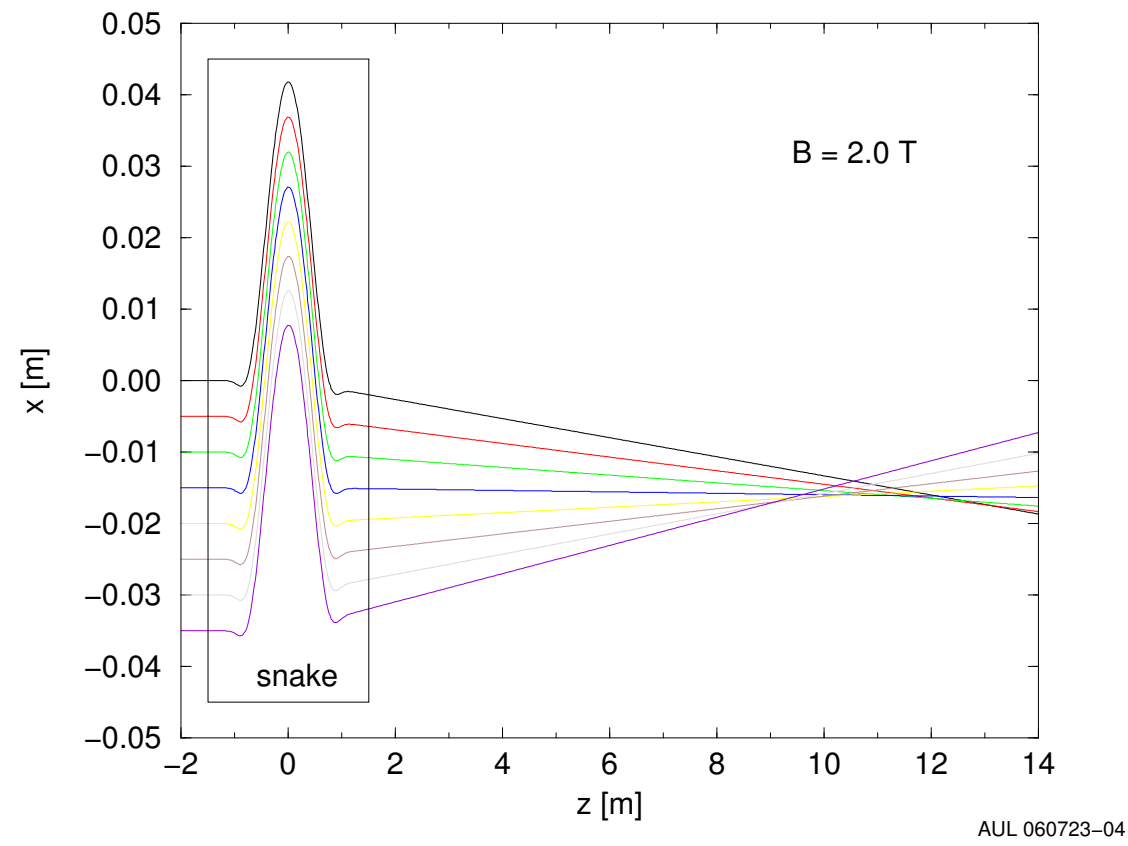

Figure 1: Cold snake. $\mathrm{B}=2 \mathrm{~T}$, Horizontal trajectories for particles injected at various $\mathrm{x}$-offset positions, and $\mathrm{y}=0 . \gamma=2.5$.

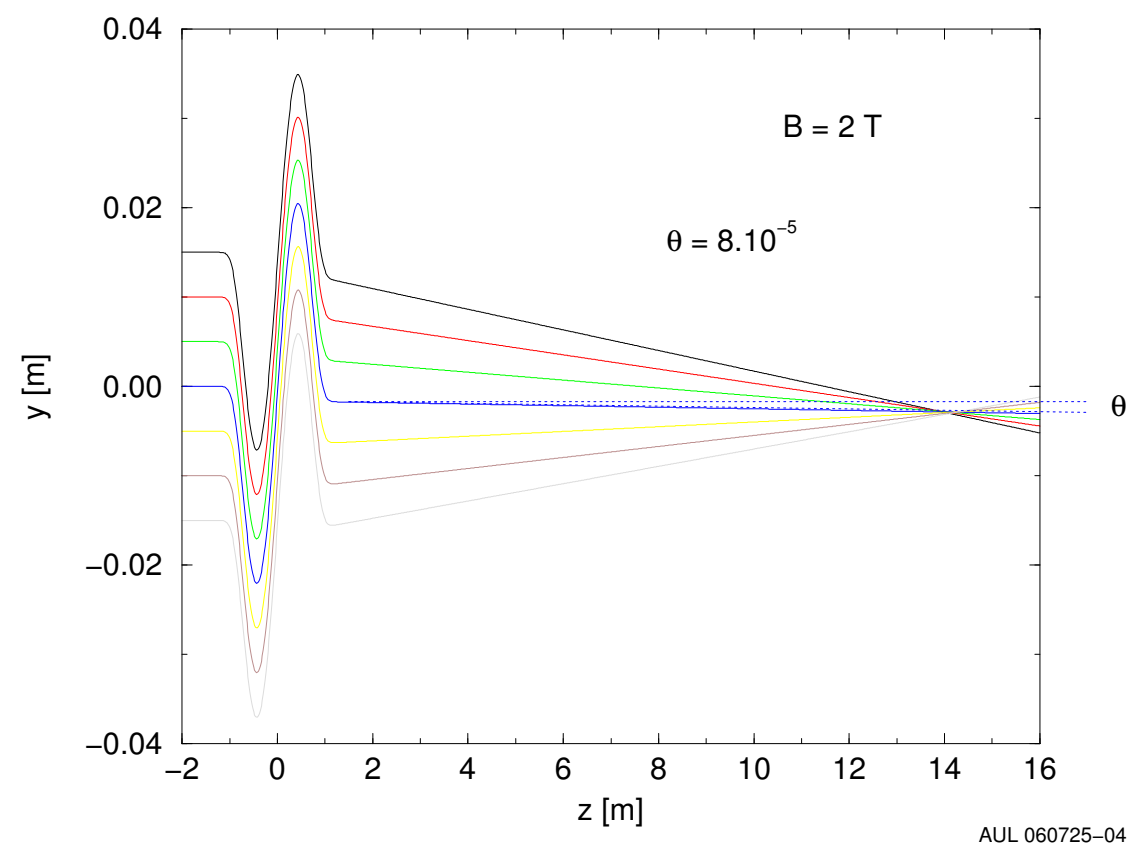

Figure 2: Cold snake. $\mathrm{B}=2 \mathrm{~T}$, Vertical trajectories for particles injected at various $\mathrm{y}$-offset positions, and $\mathrm{x}=0 . \gamma=2.5$. 


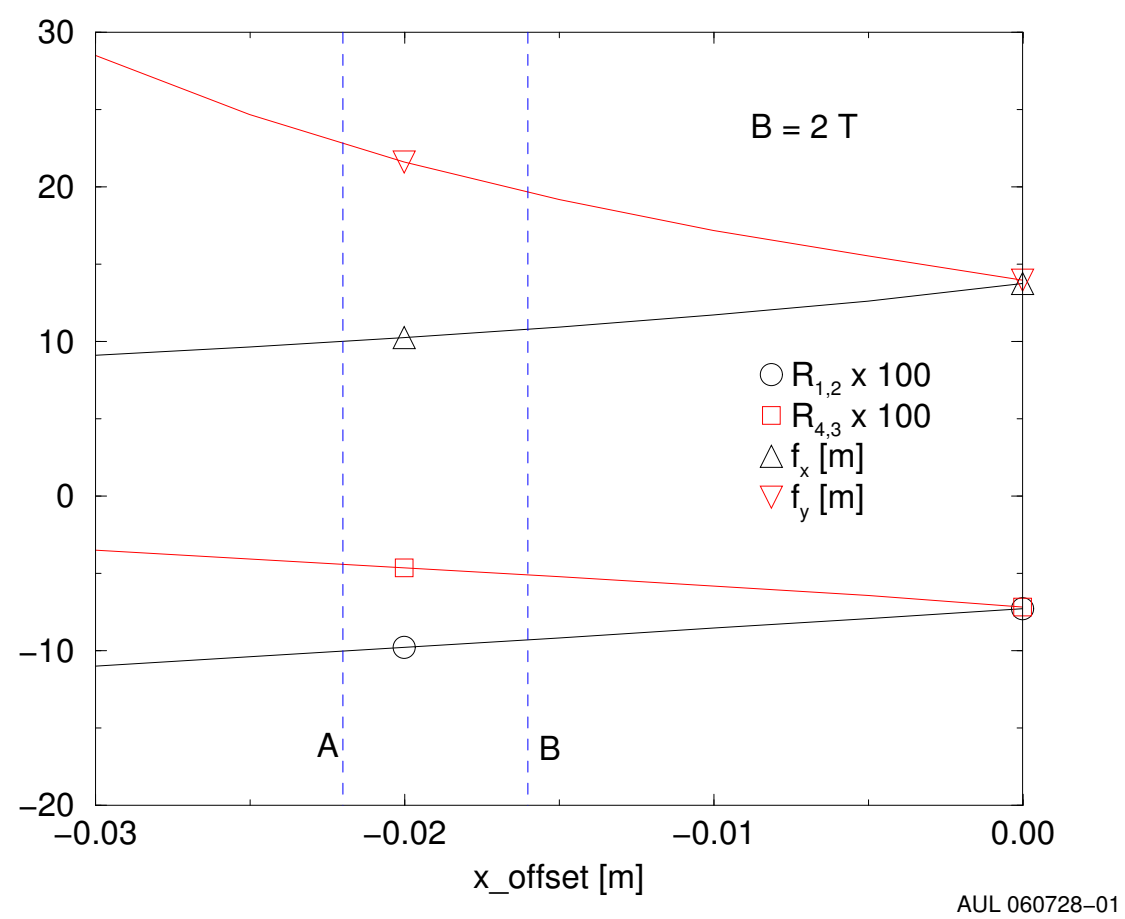

Figure 3: Cold snake. $\mathrm{B}=2 \mathrm{~T}$, Focusing matrix elemnt and focal length in the horizontal and vertical plane, respectively, vs. $\mathrm{x}$-offset of the orbit at injection. $\mathrm{y}=0$ for all curves. Dashed line 'A' marks the working offset $\mathrm{x}=22 \mathrm{~mm}$ that balances the horizontal orbit in the snake and line 'B' marks the offset $\mathrm{x}=16 \mathrm{~mm}$ that produces a straight beam at snake's exit. $\gamma=2.5$.

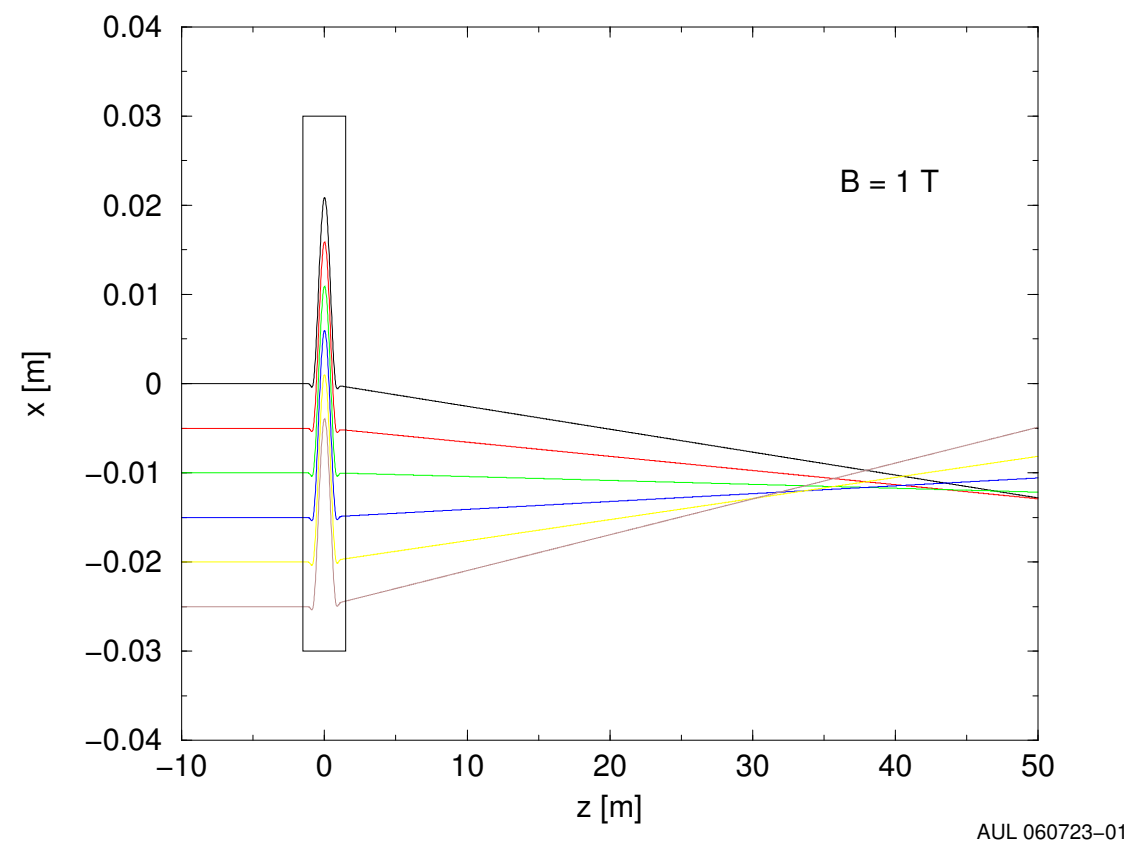

Figure 4: Cold snake. $\mathrm{B}=1 \mathrm{~T}$, Horizontal focusing at $\mathrm{B}=1 \mathrm{~T} . \gamma=2.5$. 


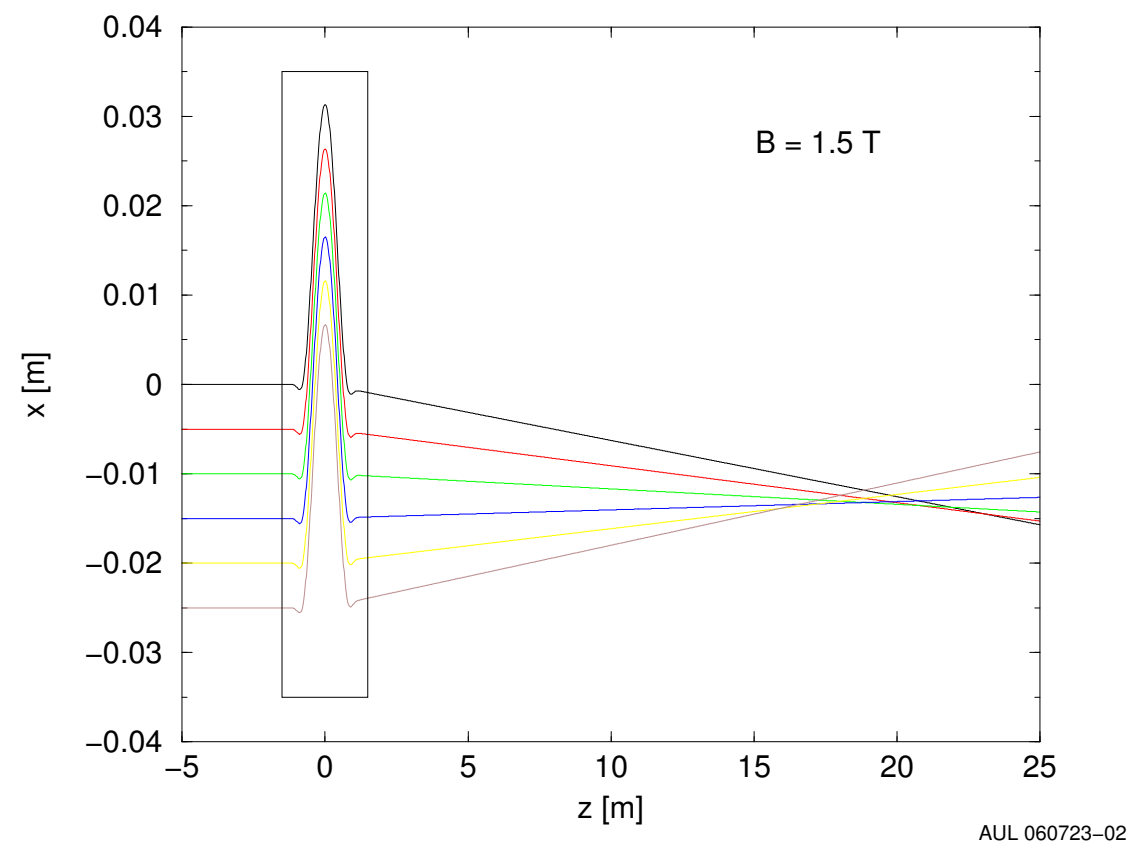

Figure 5: Cold snake. $\mathrm{B}=1.5 \mathrm{~T}$, Horizontal focusing at $\mathrm{B}=1.5 \mathrm{~T} . \gamma=2.5$.

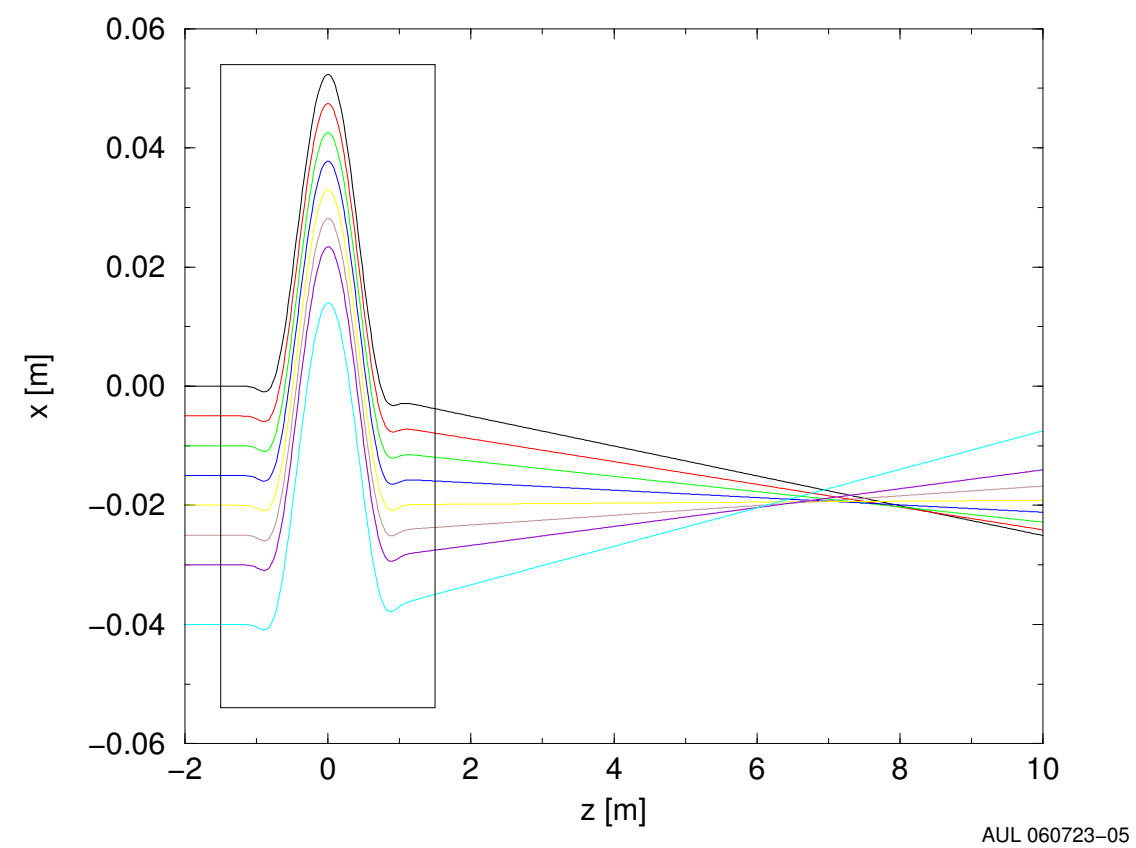

Figure 6: Cold snake. $\mathrm{B}=2.5 \mathrm{~T}$, Horizontal focusing at $\mathrm{B}=2.5 \mathrm{~T} . \gamma=2.5$. 


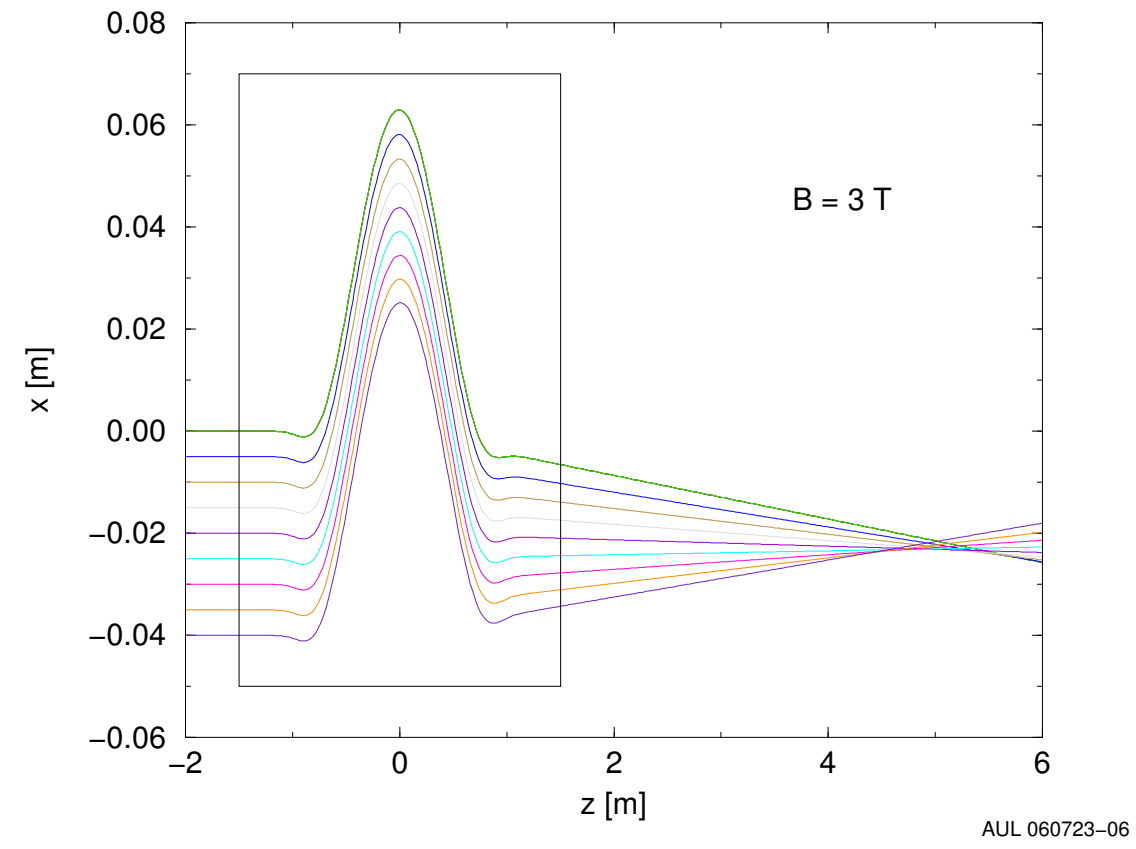

Figure 7: Cold snake. $\mathrm{B}=3 \mathrm{~T}$, Horizontal focusing at $\mathrm{B}=3 \mathrm{~T} . \gamma=2.5$.

\section{References}

[1] A.U.Luccio, R.Gupta, W.MacKay and T.Roser: Cold AGS Snake Optimization by Modeling. Technical Report C-A/AP/128, Brookhaven National Laboratory. Upton, NY, December 2003.

[2] A.U.LuCCIO: Numerical Optimization of Siberian Snakes and Spin Rotators for RHIC. In: Trends in Collider Spin Physics, Trieste, Italy, 5-8 Dec 1995, World Scientific, p.244.

[3] A.U.Luccio and T.Roser: Matching the Cold Snake to the AGS Lattice. Technical Report C-A/AP/167, Brookhaven National Laboratory. Upton, NY, September 2004. 